University of Nebraska - Lincoln

DigitalCommons@University of Nebraska - Lincoln

Faculty Publications from the Harold W. Manter Laboratory of Parasitology

10-1999

\title{
Differentiation of Mexican Species of Haematoloechus Looss, 1899 (Digenea: Plagiorchiformes): Molecular and Morphological Evidence
}

\author{
Virginia León-Règagnon \\ Universidad Nacional Autónoma de México \\ Daniel R. Brooks \\ University of Toronto, dnlbrooks@gmail.com \\ Gerardo Perez-Ponce de Leon \\ Universidad Nacional Autónoma de México, ppdleon@servidor.unam.mx
}

Follow this and additional works at: https://digitalcommons.unl.edu/parasitologyfacpubs

Part of the Parasitology Commons

León-Règagnon, Virginia; Brooks, Daniel R.; and Perez-Ponce de Leon, Gerardo, "Differentiation of Mexican Species of Haematoloechus Looss, 1899 (Digenea: Plagiorchiformes): Molecular and Morphological Evidence" (1999). Faculty Publications from the Harold W. Manter Laboratory of Parasitology. 284. https://digitalcommons.unl.edu/parasitologyfacpubs/284

This Article is brought to you for free and open access by the Parasitology, Harold W. Manter Laboratory of at DigitalCommons@University of Nebraska - Lincoln. It has been accepted for inclusion in Faculty Publications from the Harold W. Manter Laboratory of Parasitology by an authorized administrator of DigitalCommons@University of Nebraska - Lincoln. 


\title{
DIFFERENTIATION OF MEXICAN SPECIES OF HAEMATOLOECHUS LOOSS, 1899 (DIGENEA: PLAGIORCHIFORMES): MOLECULAR AND MORPHOLOGICAL EVIDENCE
}

\author{
Virginia León-Règagnon, Daniel R. Brooks*, and Gerardo Pérez-Ponce de León \\ Laboratorio de Helmintología, Instituto de Biología, Universidad Nacional Autónoma de México, Apartado Postal 70-153, CP 04510 México, \\ D.F., México
}

\begin{abstract}
Molecular evidence is interpreted in the light of morphology to examine the validity of several species of Haematoloechus described as Mexican endemics. Internal transcribed spacers 1 and 2 and $28 \mathrm{~S}$ ribosomal genes were sequenced for 11 isolates. Phylogenetic analysis of separate partitions and combined databases was conducted. Results were analyzed, in the light of morphological evidence. Haematoloechus macrorchis is proposed as a junior synonym of Haematoloechus longiplexus. Haematoloechus pulcher is a sibling species with Haematoloechus complexus in Lerma wetlands. In Mexico, Haematoloechus medioplexus is distributed along the east coast coinciding with the distribution of Rana berlandieri. The sister species of $H$. medioplexus is Haematoloechus coloradensis, sharing the distribution of the uterus as a synapomorphic character. Haematoloechus illimis is more closely related to $H$. medioplexus and $H$. coloradensis than to $H$. complexus. It can be distinguished by the distribution of the uterus, lobed ovary, and testes.
\end{abstract}

The members of Haematoloechus Looss, 1899 represent 1 of the most common and characteristic groups of digeneans inhabiting anurans. More than 50 species have been described worldwide, all living as adults in anuran lungs. Nine species have been reported from Mexico (Caballero and Sokoloff, 1934; Caballero, 1941, 1942a, 1942b; Bravo, 1943; Martínez, 1969; Guillén-Hernández, 1992; León-Règagnon, 1992; Pulido, 1994), 5 of which have been named as distinct species endemic in the central plateau. The morphological characters used to differentiate these species from those previously described are problematic (Prokopic and Krivanec, 1974; Kennedy, 1980a, 1980b, 1981), and the validity of some of them is doubtful. DNA sequences represent a relatively new and potentially valuable source of data to help solve taxonomic and phylogenetic problems involving parasitic platyhelminths (Blair et al., 1996; McManus and Bowles, 1996). In the present study, we sequenced the ribosomal internal transcribed spacers (ITS1 and ITS2) and the D1 variable region of the 28s gene for 7 nominal species of Haematoloechus from Mexico and the U.S.A., following suggestions that these regions would be informative at the scale of closely related species (Luton et al., 1992; Barker et al., 1993). We used a combination of the new molecular data and reassessment of the morphological features of nominal taxa to examine the validity of several of the species of Haematoloechus described as Mexican endemics.

\section{MATERIALS AND METHODS}

We collected tissue samples during 1996 and 1997 (Table I summarizes collecting localities and hosts). Host and parasite tissues are deposited in the frozen tissue collection of the Zoology Department, Institute of Biology, Universidad Nacional Autónoma de México (UNAM). Worms were allocated to morphospecies in vivo, using morphological characters suggested in the original descriptions (Stafford, 1902; Krull, 1933; Caballero, 1941, 1942b; Bravo, 1943). Voucher specimens were relaxed in hot tap water, fixed with alcohol-formalinacetic acid or Bouin's fluid, and stored in $70 \%$ ethanol before being stained with Mayer's paracarmine, Ehrlich's hematoxylin, or Gomori's trichrome and mounted in Canada balsam as whole mounts for comparison with specimens from the Colección Nacional de Helmintos (CNHE), Instituto de Biología, UNAM, from the U.S. National Parasite

Received 2 October 1998; revised 20 March 1999; accepted 20 March 1999.

* Department of Zoology, University of Toronto, Canada. 25 Harbord St. Toronto, Ontario, Canada M5S 3G5.
Collection (USNPC), Beltsville, Maryland, and from the Harold W. Manter Laboratory (HWML), University of Nebraska State Museum. Samples for molecular work were preserved in absolute ethanol.

Five species previously recorded in Mexico were identified using morphological characters in this study, e.g., Haematoloechus coloradensis Cort, 1915, Haematoloechus complexus (Seely, 1906) Krull, 1933, Haematoloechus illimis Caballero, 1942, Haematoloechus macrorchis Caballero, 1941, and Haematoloechus pulcher Bravo, 1943. Additionally, 2 specimens were collected from Rana vaillanti Brocchi, 1877 in Los Tuxtlas, Veracruz, whose specific identity could not be established using morphological characters due to poor preservation. Sequences of these species and specimens of Haematoloechus longiplexus Stafford, 1902 and Haematoloechus medioplexus Stafford, 1902 collected in Nebraska, U.S.A. were compared. Worms were dissected to remove host blood from the ceca. When possible, more than 1 sample was sequenced to assess intraspecific variation. Frog tissue was processed for molecular work for comparison and to ensure that the source of the DNA was worms tissues. Standard phenol extraction methods were used to recover DNA from entire worms (a single specimen whenever possible). Laboratory protocols follow Palumbi (1996) and Hillis et al. (1996). Polymerase chain reaction was used for amplifying the DNA sample; parameters and settings follow manufacturer's recommendations and Palumbi (1996). Sequencing used Thermo Sequenase radiolabeled terminator cycle sequencing kits (Amersham Life Science, Inc., Cleveland, Ohio). Protocols follow manufacturer's recommendations with minor modifications. Amplification and sequencing of the 5' ending of the $28 \mathrm{~S}$ ribosomal gene (including the D1 variable region) was performed using the primers 28Sy 5'CTA ACC AGG ATT CCC TCA GTA ACG GCG AGT3' (forward) and 28Sz 5'AGA CTC CTT GGT CCG TGT TTC AAG AC3' (reverse) (Hillis and Dixon, 1991). The ITS1 and 5.8S ITS2 regions were amplified using the primers BD1 5'GTC GTA ACA AGG TTT CCG TA3' (forward) and BD2 5'TAT GCT TAA ATT CAG CGG GT3' (reverse) (Luton et al., 1992). Position of genes in the sequence was obtained from the alignment with the ITS1-5.8S-ITS2 sequence of Echinostoma revolutum (Froelich, 1802) Looss, 1899 (Morgan and Blair, 1995) and the sequence of D1 variable domain of the $28 \mathrm{~S}$ in Schistosoma spp. Weinland, 1858 (Barker and Blair, 1996). Sequences are available in GenBank (accession nos. AF133104-AF133114 and AF133186-AF133196).

Clustal W (Thompson et al., 1994) was used with default settings for sequence alignment. The aligned sequences were subsequently edited in ESEE (version 3; Cabot and Beckenbach, 1989). Minor modifications were made by eye to correct the computer-aligned sequences. To evaluate the phylogenetic content of the data sets, we obtained the g1 statistic as suggested by Hillis and Huelsenbeck (1992). These calculations and phylogenetic analyses were performed using PAUP (version 3.1.1; Swofford, 1993) and McClade 3.04 (Maddison and Maddison, 1992). We treated gaps either as missing data or as a fifth base; for both options, we performed exhaustive searches for the independent data sets (ITS1, ITS2, and 28S), and for the combined data set. The $5.8 \mathrm{~S}$ sequences were only used as a reference for alignment and not used in 
TABLE I. Host and locality of isolates of Haematoloechus spp. collected in Mexico and the U.S.A.

\begin{tabular}{llll}
\hline \multicolumn{1}{c}{ Isolate } & \multicolumn{1}{c}{ Helminth species } & \multicolumn{1}{c}{ Host } & \multicolumn{1}{c}{ Locality } \\
\hline Complexus1 & Haematoloechus complexus & Rana montezumae Baird, 1854 & Ciénaga de Lerma, Estado de México \\
Complexus2 & H. complexus & R. montezumae Baird, 1854 & Ciénaga de Lerma, Estado de México \\
Coloradensis & Haematoloechus coloradensis & Rana montezumae & Ciénaga de Lerma, Estado de México \\
Coloradensis* & H. coloradensis & Rana dunni Zweifel, 1957 & Pátzcuaro, Michoacán, México \\
Illimis & Haematoloechis illimis & R. montezumae & Ciénaga de Lerma, Estado de México \\
Longiplexus & Haematoloechus longiplexus & Rana catesbeiana Shaw, 1802 & Genoa, Nebraska, U.S.A. \\
Macrorchis & H. longiplexus & R. montezumae & Ciénaga de Lerma, Estado de México \\
Medioplexus & Haematoloechus medioplexus & Rana pipiens Schreber, 1782 & Holt Creek, Nebraska, U.S.A. \\
Pulcher1 & H. complexus & Ambystoma lermaensis Taylor, 1940 & Ciénaga de Lerma, Estado de México \\
Pulcher2 & Haematoloechus pulcher & A. lermaensis & Ciénaga de Lerma, Estado de México \\
Tuxtlas1 & H. medioplexus & Rana vaillanti Brocchi, 1877 & Los Tuxtlas, Veracruz, Mexico \\
Tuxtlas2 & Haematoloechus sp. & R. vaillanti & Los Tuxtlas, Veracruz, Mexico \\
\hline
\end{tabular}

* This isolate was not included in the analysis because only the $28 \mathrm{~S}$ gene sequence was obtained, and no variation with respect to the isolate from $R$. montezumae was found.

phylogenetic analyses. Bootstrap resampling was conducted with 1,000 replicates in the branch and bound option.

\section{RESULTS}

A total of $1,836 \mathrm{bp}-542 \mathrm{bp}$ of the $5^{\prime}$ end of the ITS1 (incomplete), $124 \mathrm{bp}$ of the $5.8 \mathrm{~S}$, the entire ITS 2 (287 bp), and $883 \mathrm{bp}$ of the $5^{\prime}$ end of the $28 \mathrm{~S}$ (Fig. 1) - was sequenced and aligned for 7 species of Haematoloechus (11 isolates).

The sequenced region of the ITS 1 exhibited $16 \%$ variability (87 variable sites, not including gaps). In the case of $\mathrm{H}$. $\mathrm{ma}$ crorchis and $H$. longiplexus, there are 3 inserts: the first is 9$15 \mathrm{bp}$ long in position 42 , the second is $47 \mathrm{bp}$ long in position 127 and is repeated 3 consecutive times, and the third is $4 \mathrm{bp}$ long in position 277 (Fig. 1). ITS2 shows a higher variability (without considering inserts), with $22.3 \%$ of variable sites (64 out of 287); an insert of 17 bp can be seen in $H$. complexus, $H$. pulcher, $H$. macrorchis, and $H$. longiplexus close to the $5^{\prime}$ end of the molecule. The sequenced region of the $28 \mathrm{~S}$ is $15.4 \%$ variable (136 variable sites) (Fig. 1). A distance matrix is shown in Table II.

The $\mathrm{g} 1$ statistic values are -2.82 for the ITS 1 data, -0.65 for the ITS $2,-0.79$ for the $28 \mathrm{~s}$, and -1.39 for the combined data set, showing that the data sets are significantly more structured than random data $(P=0.01)$ (Hillis and Huelsenbeck, 1992).

Tree topology was not affected considering gaps as missing data or as a fifth base. Phylogenetic analyses of each separate data set gave similar topologies for most of the tree, with the exception of pulcher 2 and Tuxtlas 2 , whose position varies when analyzing ITS 1 and ITS2, respectively. Consistency indexes and bootstrap values are shown in Figure 2. Following the methodology suggested by Wiens (1998), we combined the 3 data sets that resulted in a single most parsimonious tree with 756 steps and a confidence interval (CI) of 0.84 (430 steps; CI $=0.82$ when gaps were treated as missing data). High bootstrap values were obtained for each node and are indicated on the tree (Fig. 3). Haematoloechus longiplexus and H. macrorchis group together in all cases; the same happens with $H$. complexus, and pulcher1, and with $H$. medioplexus, Tuxtlas $1, H$. coloradensis, and $H$. illimis.

\section{DISCUSSION}

Internal transcribed spacers have been used to help reconstruct phylogenetic relationships among closely related helminth groups. ITS 1 has been shown to be relatively conservative but has several repeated units that are responsible for its length variation, even among closely related species (Luton et al., 1992; Kane and Rollinson, 1994). Bowles et al. (1995) found divergent paralogues of ITS1 in Echinococcus Rudolphi, 1801 (Cestoda), a feature that is very common in plants (Buckler et al., 1997). We did not find divergent paralogues in Haematoloechus ITS1, but we did find them in frogs' DNA. Large repeating units are present in the inserts of $H$. macrorchis and $\boldsymbol{H}$. longiplexus sequences, as reported for other genera of digeneans, e.g., Dolichosaccus Johnston, 1912 (Luton et al., 1992) and Schistosoma (Kane and Rollinson, 1994), although in the species of Echinostoma Rudolphi, 1809 no inserts have been found (Morgan and Blair, 1995). The insertion of large sequences in ITS 1 seems to be a feature that appears independently in unrelated groups. This feature makes ITS 1 only suitable for phylogenetic studies at the species or populations level. ITS2 has been reported to vary from $1.1 \%$ in closely related species of Schistosoma (Kane and Rollinson, 1994) to $25.87 \%$ in distantly related species in the same genus (Bowles et al., 1995). We found a similar amount of variation among Haematoloechus spp. (22.3\%). The D1 region of the $28 \mathrm{~S}$ gene has been used in phylogenetic studies at different taxonomic levels, from species of the same genus (Littlewood and Johnston, 1995; Barker and Blair, 1996) to species from different families in a class (Barker et al., 1993). The region we used in this study includes the variable D1, and we found it to be more conservative than the ITS 1 and 2 but still variable enough to obtain some phylogenetic information, e.g., $>10 \%$ (Hillis and Dixon, 1991).

The phylogenetic hypotheses obtained from each data set differ in the position of pulcher2 and Tuxtlas 2 . Whereas in the ITS 1 and ITS2 hypotheses, one or the other were included in the complexus group (complexus $1+$ complexus $2+$ pulcher 1 ); in the $28 \mathrm{~S}$ hypothesis both were included in this group (Fig. 2). The conflicting nodes were strongly supported in each case. There are 2 possible explanations for the difference in the 
ITS1

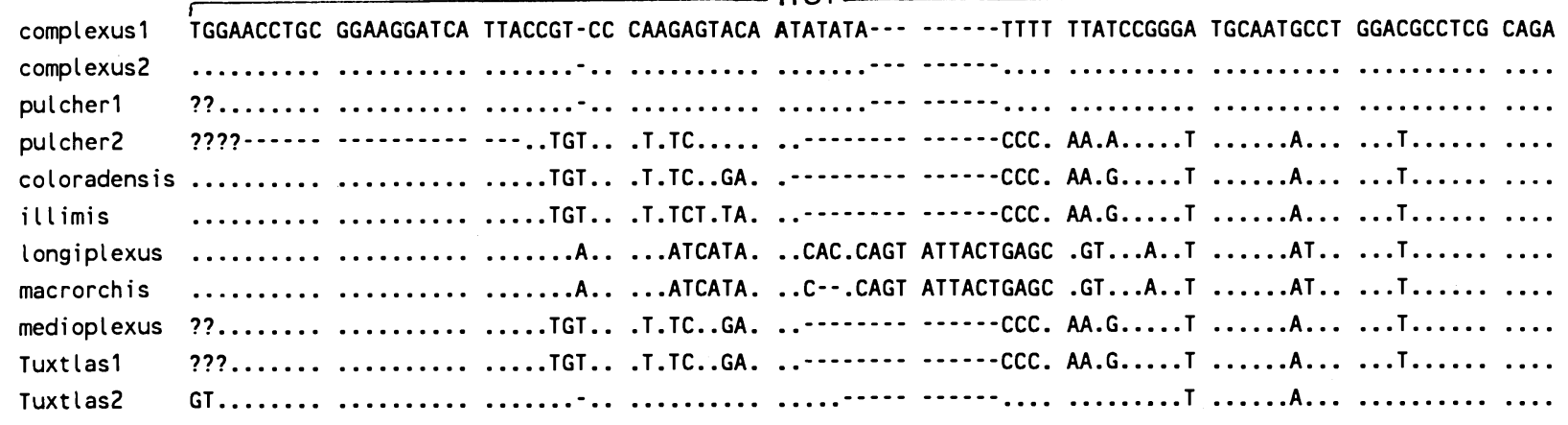

complexus 1 complexus2 pulcher 1 pulcher2 coloradens is illimis longiplexus macrorch is medioplexus Tuxtlas 1 Tuxtlas2

complexus 1 complexus2 pulcher 1 pulcher2 coloradensis illimis longiplexus macrorch is medioplexus Tuxtlas 1 Tuxtlas2

complexus 1 complexus2 pulcher 1 pulcher2 coloradensis illimis longiplexus macrorch is medioplexus Tuxtlas 1 Tuxtlas2

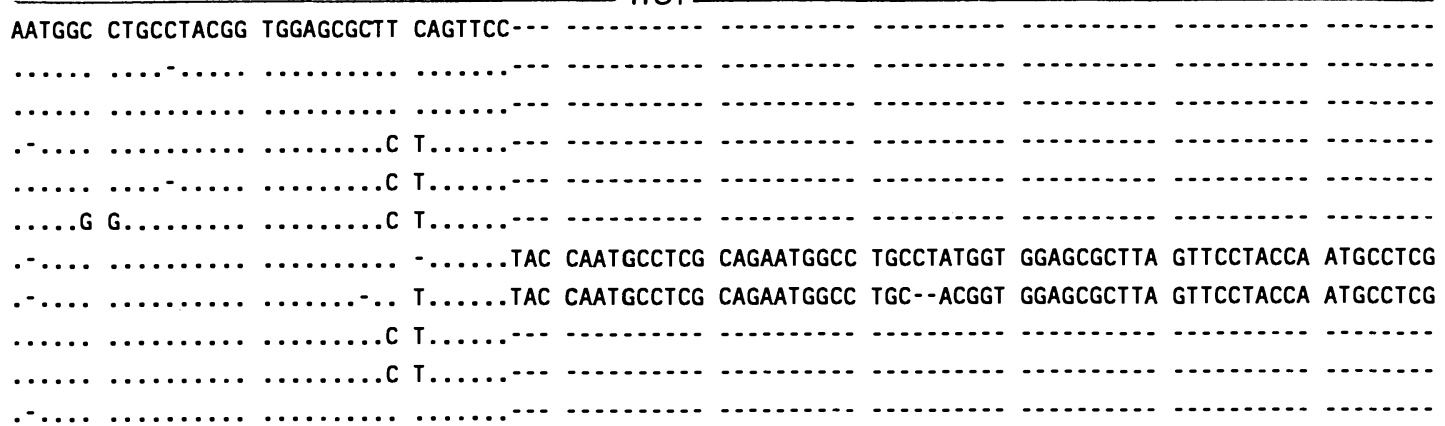

ITS1

GGCTGTACC TGGTGCATTC GTGTGCCTAC GTGCAGTC-- CCCTATGACA GGGTGCCTAC TCGTCTGATG CTCGTGGGGT GCTAGCAATC TTGTA 375

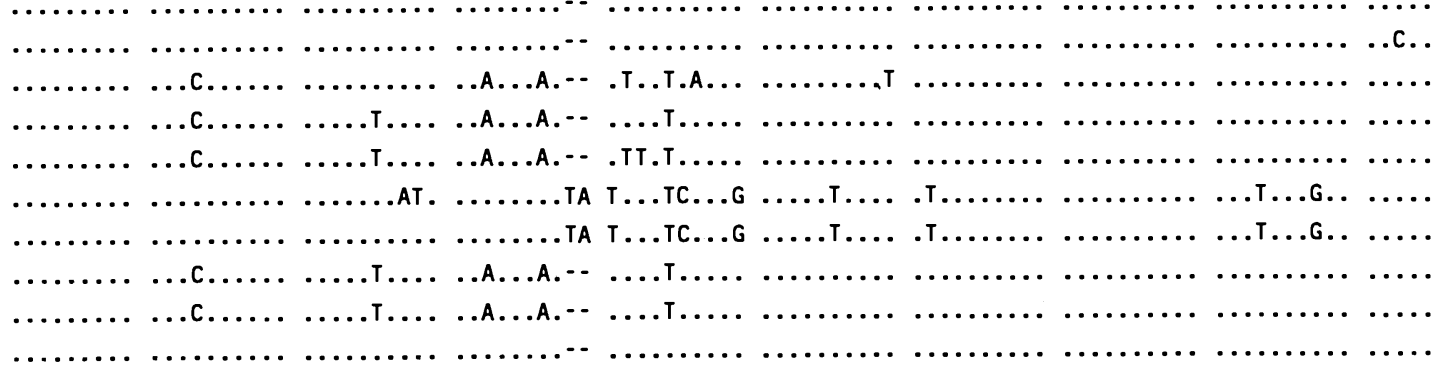

FIGURE 1. Sequences of ITS1 (partial), 5.8S (excluded from analyses), ITS2, and 28S (partial) ribosomal genes of 11 isolates of Haematoloechus species from Mexico and U.S.A. For hosts and collecting localities, see Table I. $\mathbf{O}=$ same state as in the top sequence; $-=$ gap; ? = unknown state. 
ITS1

complexus 1 complexus2 pulcher 1 pulcher2 coloradensis illimis longiplexus macrorchis medioplexus Tuxtlas 1 Tuxtlas2
TTGTC AGTCCACCTT GTGAGCGACG A??TGTGCTG TCGTTCGCGG CAGTGCTAGG CTTAAAGAGT GGTTGATTGC -ACGGCATAG TCACCGCCC 469 [....................... GC. ...................... .GTA

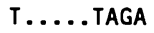

ITS1

compl exus 1

complexus2

pulcher1

pulcher2

coloradensis

illimis

longiplexus

macrorchis

medioplexus

Tuxtlas 1

Tuxtlas2

\section{T GTTAAATTGT ITACAAAACC - TITTACACT GTTCAAGTGG ITCAGATCAG CCTCGGTTGG ITTGGATCAT TG}

$5.8 S$

GTACGATG AAGAGCGCC 559

.

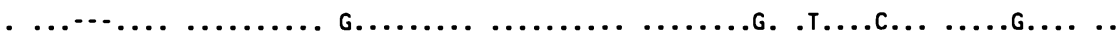

. .............. G..................... .т..............

. .....................................................

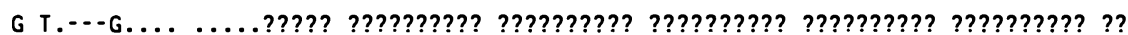

G T...GT.... ...... CTA ????????? ????????? ????????? ????????? ????????? ??

. ............... G.....................G. .т...............

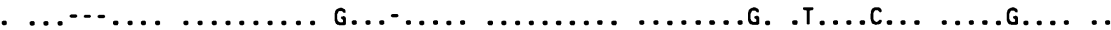

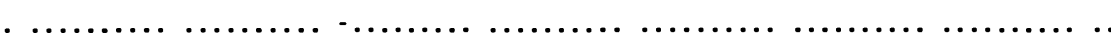

...............

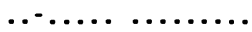

.............A

-...............

.-.GTGGA G........

...............

................

............A

$\ldots \cdots \ldots \ldots \ldots A$

...........A

$5.8 S$

compl exus 1

complexus2

pulcher 1

pulcher2

C AGAACTGTGT GAATTAATGT G--AACTGCA TACTGCTTTG

ACATCGACA TCTTGAACGC ATATTG?GCC ATGGGTTATC ?C??G-...- CC 652

coloradensis

illimis

$\cdot$

- .......

-.............

.

longiplexus

macrorchis

medioplexus

Tuxtlas 1

CC..

$\cdots \cdots \cdots$

.................

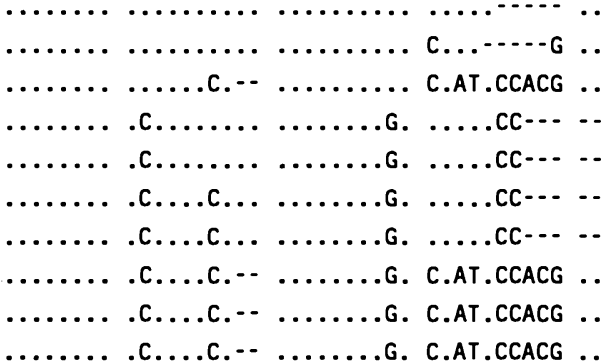

Tuxtlas2

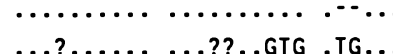

T

$\mathrm{T} . \mathrm{.}$

.G. C.AT.CCACG ..

ITS2

\begin{tabular}{|c|c|}
\hline compl exus 1 & TGTCCGAG \\
\hline compl exus2 & \\
\hline pulcher1 & \\
\hline ulcher2 & \\
\hline oloradens is & $\ldots \ldots$ \\
\hline llimis & \\
\hline ongiplexus & ....... \\
\hline acrorchis & \\
\hline dioplexus & \\
\hline axtlas1 & \\
\hline uxtlas2 & \\
\hline
\end{tabular}

FIGURE 1. Continued. 
ITS2

complexus 1 complexus2 pulcher1 pulcher2 coloradens is

ill imis longiplexus macrorchis medioplexus Tuxtlas Tuxtlas2

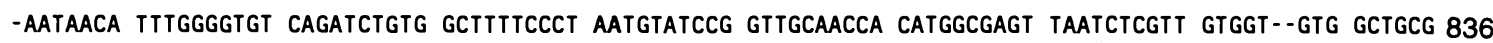
-

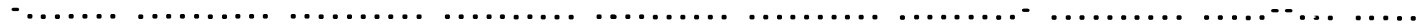

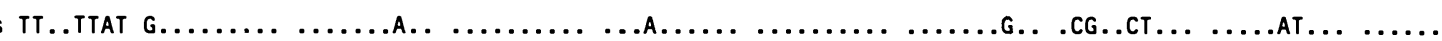
ТТ..ТТАТ G.......................................................... АT..T.АT .................... ..................G.A ATT........GT.....T.

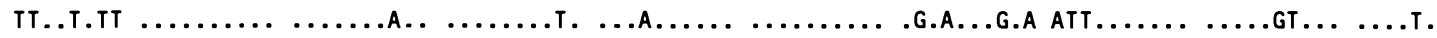
TT..TTAT G.........................................CG..СT............. ТT.АTTGT G....................................................... ТТ..ТТАТ G........................................................

ITS2

complexus 1 compl exus2 pulcher 1 pulcher2 coloradens is illimis longiplexus macrorchis medioplexus Tuxt las 1 Tuxtlas2

compl exus1
compl exus2
pulcher1
pulcher2
coloradens is
illimis
longiplexus
macrorchis
medioplexus
Tuxt las1
Tuxt las2

complexus 1 complexus2 pulcher 1 pulcher2 coloradens is illimis longiplexus macrorch is medioplexus Tuxtlas 1 Tuxtlas2

\section{GAGT CGTGGCTCAA TTGGTTGATT --A ATGTGCG CGCTCCGTCA GTTCACTCGT GTTGTTAACC AAGATTGGCG TATCTACGTC AATGTTATTC 930}

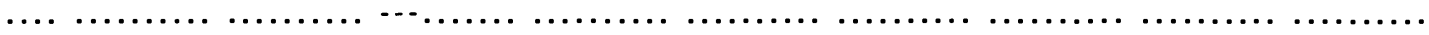

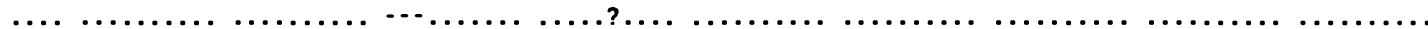

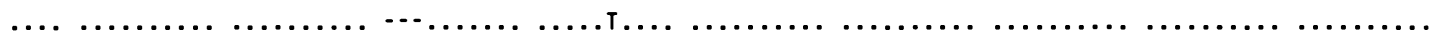
$\ldots \ldots \ldots \ldots \ldots \ldots$. . . .

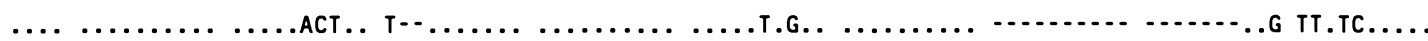

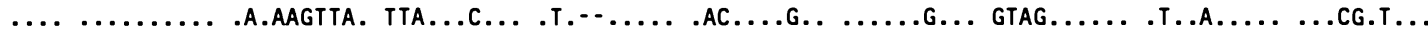

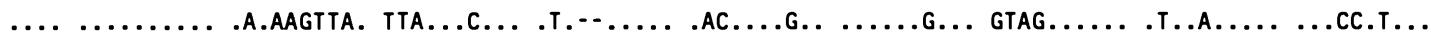

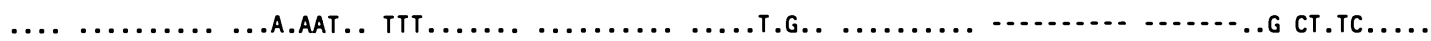

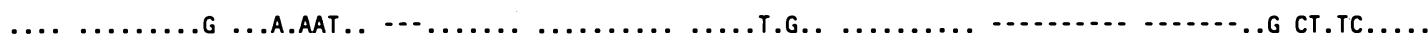

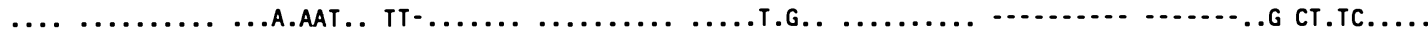

ITS2 CTGACCTCGG ATCAGACGTG AAT ................ $\ldots \ldots \ldots \ldots \ldots \ldots \ldots$ [.....................

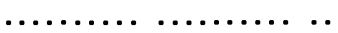

$\ldots \ldots \ldots \ldots \ldots \ldots \ldots \ldots$ [.................... [................... [.................... $\ldots \ldots \ldots \ldots \ldots \ldots \ldots \ldots$ $\ldots \ldots \ldots \ldots \ldots \ldots \ldots \ldots$
285

GGGATAA GCCCAGCACC GAAGCCTGTA GCCATTTGGT TACTAGGCAA TGTGGTGTTT AGGTCGTTCC 1020

С.

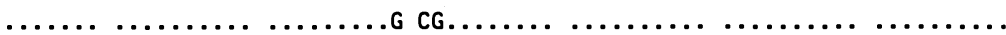

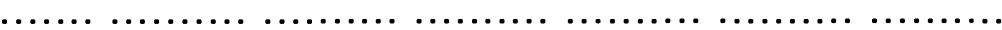

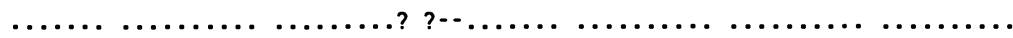
$\ldots \ldots$. $\ldots \ldots \ldots \ldots \ldots \ldots \ldots \ldots$ ? ${ }^{\prime}, \ldots \ldots \ldots \ldots \ldots \ldots \ldots \ldots \ldots \ldots \ldots \ldots \ldots \ldots$

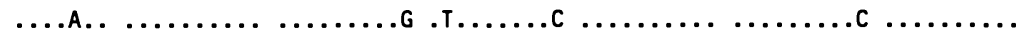

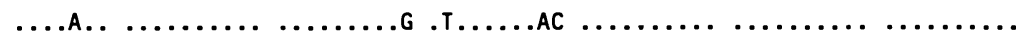

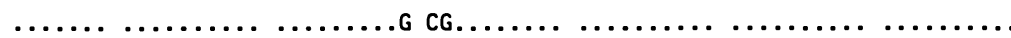

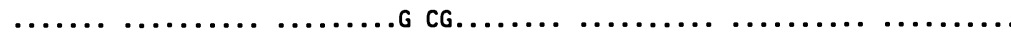

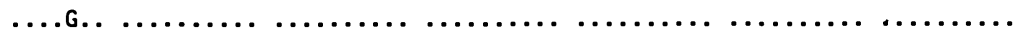

28S

GTGGATATTC TGCTCCACCC TAAGTCC-AT CAATGAGTAC GGTATTAT-G GACATGGCCC ATAGAGGGTG AAAGGCCCGT GGGGGTGGG ATT 1113

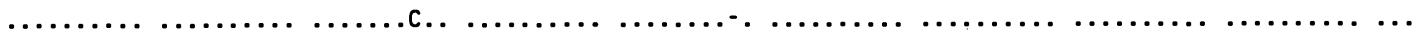

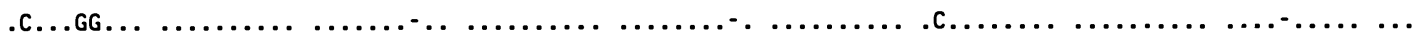
$\ldots \ldots \ldots \ldots \ldots \ldots \ldots \ldots \ldots \ldots \ldots \ldots-\ldots \ldots \ldots \ldots \ldots \ldots \ldots \ldots \ldots{ }^{\prime} \ldots \ldots \ldots \ldots \ldots \ldots \ldots \ldots \ldots \ldots \ldots \ldots \ldots \ldots \ldots \ldots \ldots \ldots \ldots$

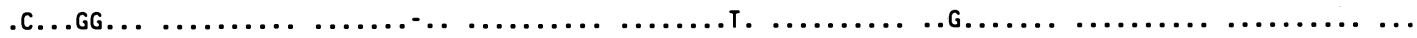

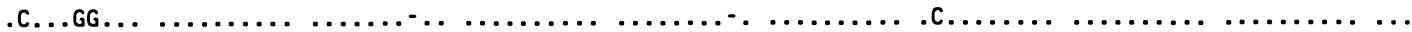

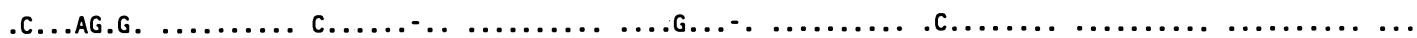

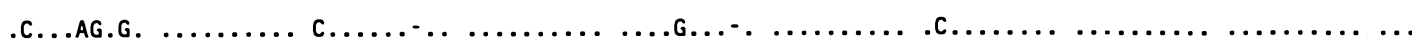

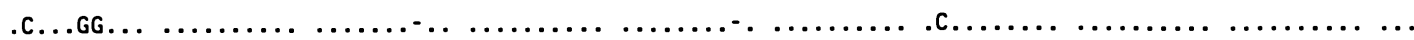

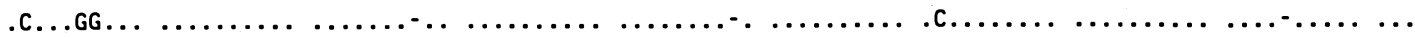

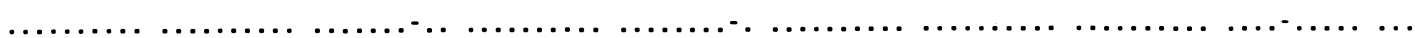

FIGURE 1. Continued. 
complexus 1 compl exus2 pulcher 1 pulcher2 coloradens is illimis longiplexus macrorchis medioplexus Tuxtlas 1 Tuxtlas2

complexus 1 complexus2 pulcher 1 pulcher2

coloradensis. illimis longiplexus macrorchis medioplexus Tuxtlas 1 Tuxtlas2

complexus 1 complexus2 pulcher1 pulcher2 coloradens is illimis longiplexus macrorchis medioplexus Tuxtlas 1 Tuxtlas2

complexus 1 complexus2 pulcher 1 pulcher2 coloradens is illimis longiplexus macrorchis medioplexus Tuxtlas 1 Tuxtlas2
$28 S$

CGGTAGG ACAGAATATT CTTGGGTAGA CCTTGGAGTC GGGTTGTTTG TGAATOCAGC CCAAAGTGG TGGT-AAACT CCATCCAAGG CTAAATA 1207

FIGURE 1. Continued. 
28S

complexus 1 complexus2 pulcher 1 pulcher2 coloradensis longiplexus macrorch is medioplexus Tuxt las 1 Tuxtlas2 illimis

TC AGAGTGTTCA CCACGACCGG CGCCGCTGTC TGGCCTCTAT AGtTAAACCG GTTTTGCATA GTCCTTGTGG CTTTGCTTAG TCGGGACGGC A 1581

. .........

.........

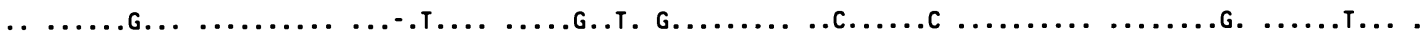

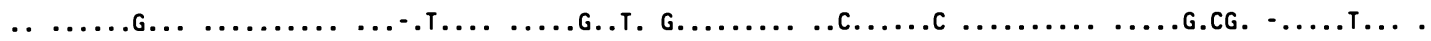

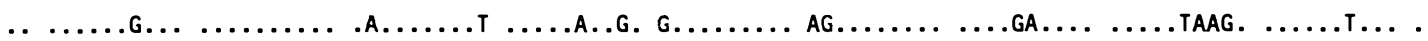

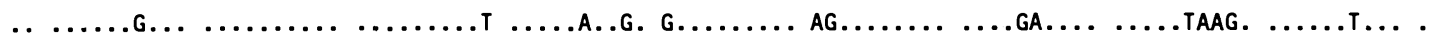

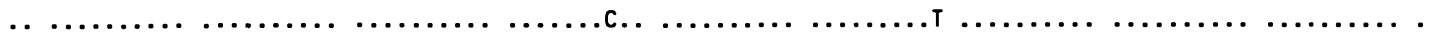

285

complexus 1

complexus2

pulcher 1

pulcher2

coloradensis

illimis

longiplexus

macrorchis

medioplexus

Tuxtlas 1

Tuxtlas2

complexus 1

complexus2

pulcher 1

pulcher2

coloradens is

illimis

longiplexus

macrorch is

medioplexus

Tuxtlas 1

Tuxtlas2

complexus 1

complexus2

pulcher 1

pulcher2

coloradens is

illimis

longiplexus

macrorch is

medioplexus

Tuxtlas 1

Tuxtlas2
GGTAGCTCG TTGACTTGCT TGTGGTTGCC TGC-AAGCGT GGTTTTCGAG TGTAATCAGC TGACTGTAGT TGTTCTGTGC AGTGTGTCGG AGACG 1675

$\ldots \ldots \ldots$
$\ldots \ldots \ldots \ldots \ldots$
$\ldots$$\ldots \ldots \ldots$

$\ldots \ldots \ldots \ldots \ldots \ldots \ldots \ldots$

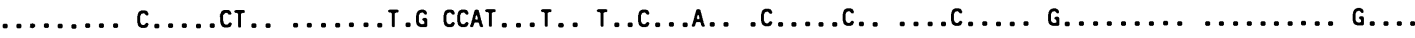

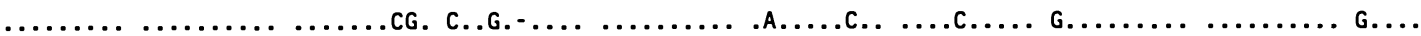

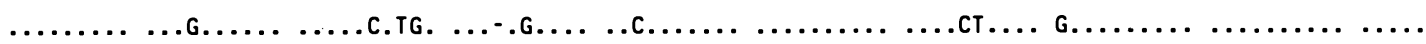

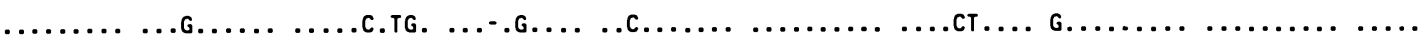

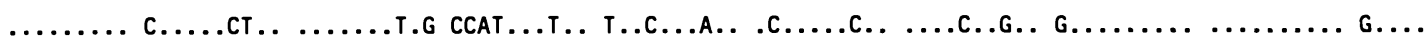

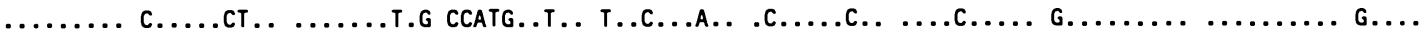

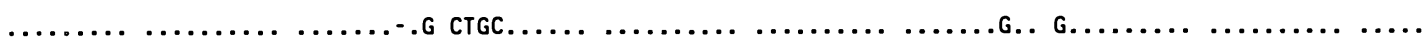

$28 \mathrm{~S}$

GCGGC TTGAGGTGTG TGCATGCGTA GTTGTTTTGC TGACTGGTTC GAGTTTGGTT ATtTGtT-GC CTGTTCATGC AGGTCTGGTA GTAGCTCGA 1769

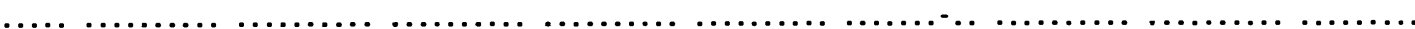

$\ldots \ldots \ldots \ldots \ldots \ldots \ldots, \ldots \ldots \ldots \ldots, \ldots \ldots \ldots \ldots, \ldots \ldots \ldots \ldots, \ldots \ldots \ldots \ldots \ldots \ldots \ldots \ldots, \ldots \ldots \ldots \ldots \ldots \ldots \ldots \ldots \ldots \ldots \ldots \ldots \ldots$

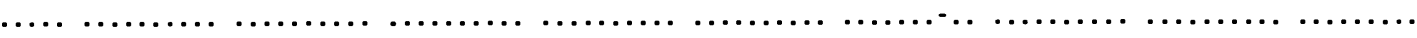

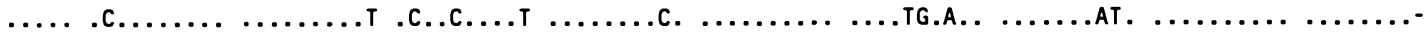

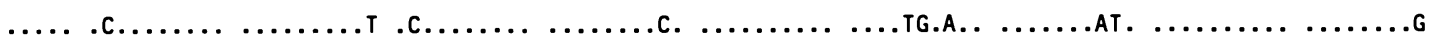

$\ldots \ldots \ldots \ldots \ldots \ldots . \ldots \ldots$ T TCG...............................AC. ...GTT... A.G...G

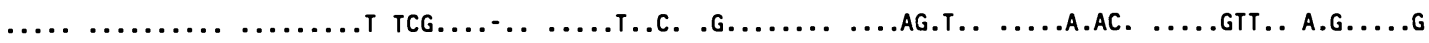

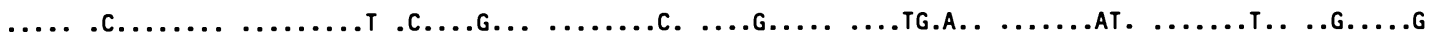

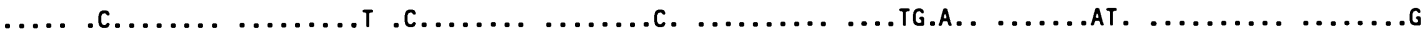

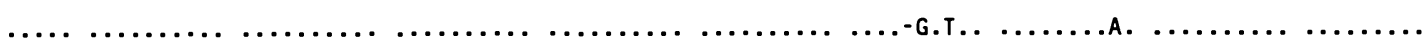

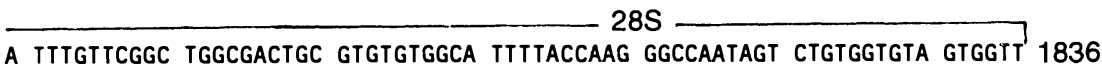

$. \ldots \ldots \ldots \ldots, \ldots \ldots \ldots \ldots, \ldots \ldots \ldots \ldots, \ldots \ldots \ldots \ldots, \ldots \ldots \ldots \ldots \ldots \ldots \ldots \ldots \ldots \ldots \ldots$

$. \ldots \ldots \ldots \ldots, \ldots \ldots \ldots \ldots, \ldots \ldots \ldots \ldots, \ldots \ldots \ldots \ldots, \ldots \ldots \ldots \ldots \ldots \ldots \ldots \ldots \ldots \ldots$

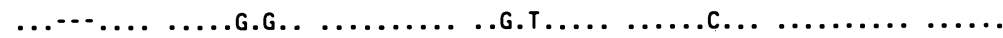

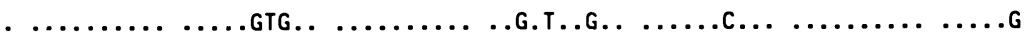

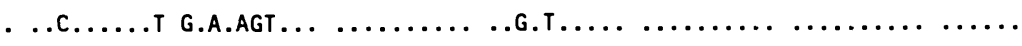

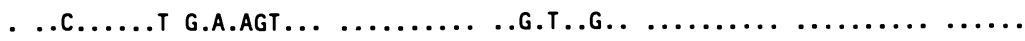

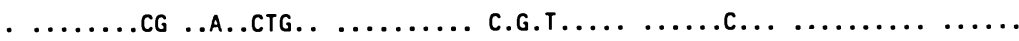

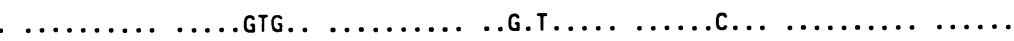

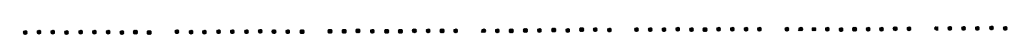

FIGURE 1. Continued. 
TABle II. Pairwise distances between taxa, calculated from the combined data set using PAUP Version 3.1.1 (Swofford, 1993).

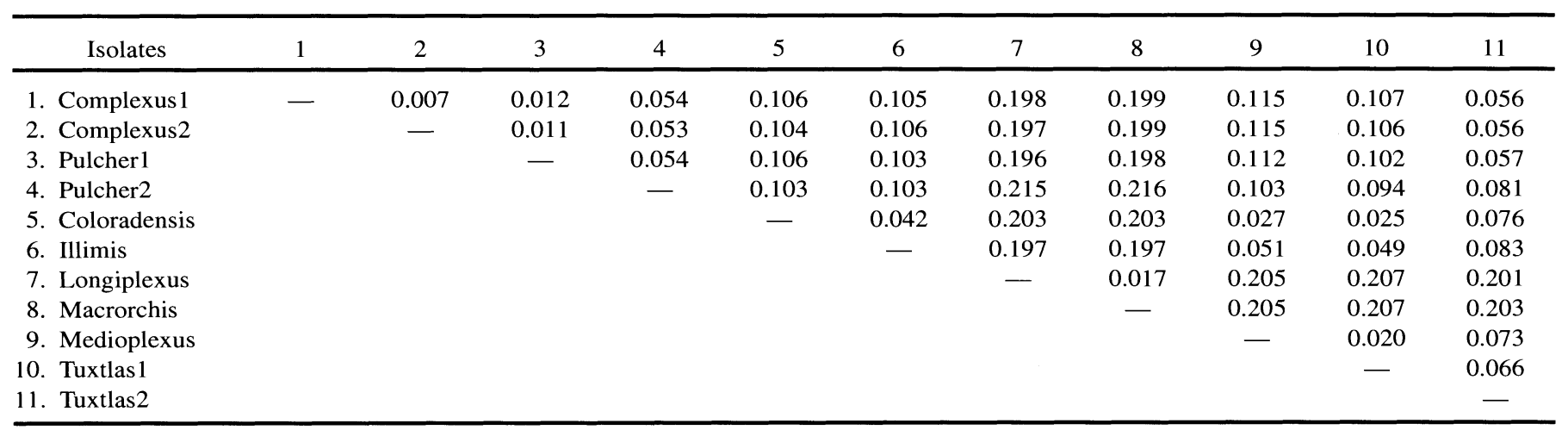

placement of those isolates. The first is that we are dealing with a case of hybridization (not with $F_{1}$ hybrids that would have both parental genomes represented [Rollinson, et al., 1990] but historical hybridization). Nevertheless, if this was the case, the isolates would be strongly associated to 1 or the other parental species, but this is not reflected in the trees (Fig. 2). The second alternative, and the one we think is the best supported by our results, is that the misplacement of these isolates is the result of noise in the data. Combining the 3 data sets allows us to increase the accuracy of the estimated tree by the use of a larger number of characters in the analysis, especially in those parts of the tree unaffected by homoplasy (Kluge, 1989; Kluge and Wolf, 1993; Wiens, 1998). The resulting hypothesis is supported by the morphological evidence. Tuxtlas 1 and pulcher 2 share with other members of the complexus group a large acetabulum, round testes and ovary, and an unordered array of uterine loops that do not go extracecal.

Specimens identified as $H$. macrorchis and $H$. longiplexus are very similar, differing by only $1.7 \%$. The phylogenetic analysis indicates that, among the taxa used in this study, $H$. longiplexus and $H$. macrorchis are each other's closest relatives. These observations could indicate that the taxa are not distinct species. As noted above, however, ITS2 has been reported to vary as little as $1.1 \%$ in closely related species of Schistosoma (Kane and Rollinson, 1994). Caballero (1941) differentiated the specimens he described as $H$. macrorchis from $H$. longiplexus by the length of the extracecal uterine loops. In the Mexican specimens, they extend anteriorly halfway between the ovary and the pharynx, whereas in $H$. longiplexus they extend anteriorly to the level of the pharynx. Caballero (1941) also reported specimens of $H$. macrorchis to have a spined tegument, whereas the tegument in $\boldsymbol{H}$. longiplexus was described as aspinose. The presence of spines by itself is a problematic character for differentiating species of this genus. Cort (1915) reported that $H$. longiplexus specimens were aspinose or spinose. Krull $(1932,1933)$ noted that in $H$. longiplexus and $H$. complexus spines can be lost during the development of the worm or with the fixation techinques, and Brooks (1976) confirmed the presence of tegumental spines on adult specimens of $H$. complexus. Manter (1938) considered Haematoloechus similiplexus Stafford, 1902 and Haematoloechus varioplexus Stafford, 1902 synonymous because the only distinguishing feature was the presence or absence of tegumental spines.

We examined specimens (CNHE 814, 815, 1555; USNPC
75446, 79466; HWML 20144, 20146, 20147, 20148, 20149, $20150,21947,22243,23255,34137)$ and found that in some specimens of $\boldsymbol{H}$. longiplexus the tegument had tiny spines, and in some specimens of $H$. macrorchis the tegument was aspinose. Likewise, the uterine loops in some specimens of $\mathrm{H}$. longiplexus reach the pharynx level, whereas in others they reach halfway between the ovary and the pharynx, as in the type specimens of $H$. macrorchis. The information obtained from reexamination of the morphology shows that the characters used originally to distinguish $H$. longiplexus and $H$. macrorchis are variable within samples purported to be one or the other. In conjunction with the low level of molecular difference, the lack of distinguishing morphological traits leads us to propose herein that $H$. macrorchis is a junior synonym of $H$. longiplexus.

The $1.7 \%$ variation between samples may indicate that they represent differentiated populations. In addition to the geographic distributions, there are some apparent differences in host species affinities. In the U.S.A. H. longiplexus is primarily a parasite of the bullfrog, Rana catesbeiana Shaw, 1802, although it has been reported with low prevalences and abundances in other frog species like Rana blairi Mecham, Littlejohn, Oldham, Brown, and Brown, 1973 and Rana pipiens Schreber, 1782 (Brooks, 1976). In Mexico, R. catesbeiana was introduced to the northern states and does not occur farther south than Zacatecas and Tamaulipas (Flores-Villela, 1993). In the present study, we found $H$. longiplexus only in Rana montezumae Baird, 1854, a member of the leopard frog clade that includes $R$. blairi and $R$. pipiens, and in very low prevalence (1.2\% in this study; Caballero [1941] reported it to be uncommon).

One of the most complex and controversial groups of nominal species of Haematoloechus are those inhabiting North American ranid frogs that have no extracecal uterine loops, spherical testes and ovaries, and distinct ventral suckers that are approximately the same size as, or slightly smaller than, the ventral sucker. Included in this group have been $H$. complexus and $H$. coloradensis in the U.S.A. east of the Rocky Mountains, Haematoloechus confusus Ingles, 1932, Haematoloechis kernensis Ingles, 1932, Haematoloechus oxyorchis Ingles, 1932, Haematoloechus tumidus Ingles, 1932, and Haematoloechus buttensis Ingles, 1936 in the west, and H. pulcher and H. illimis in Mexico.

Haematoloechus pulcher was differentiated from $H$. complexus by the presence of prominent pharyngeal glands, a rel- 


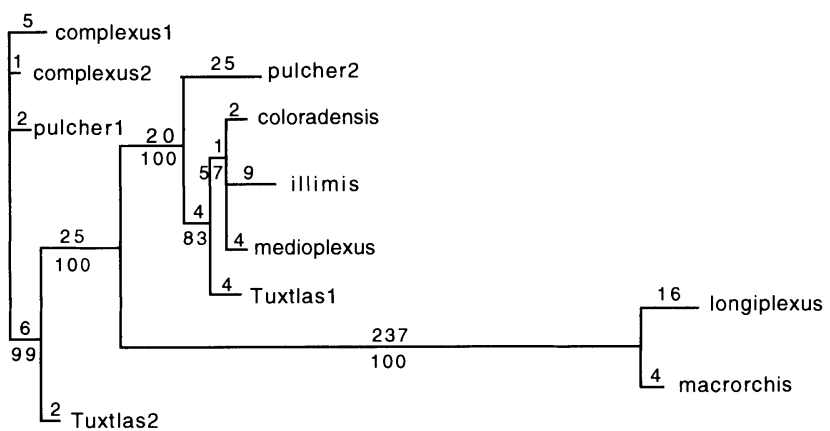

a.

b.

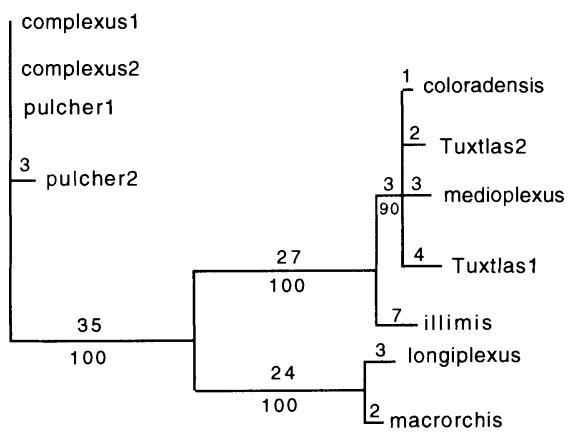

c.

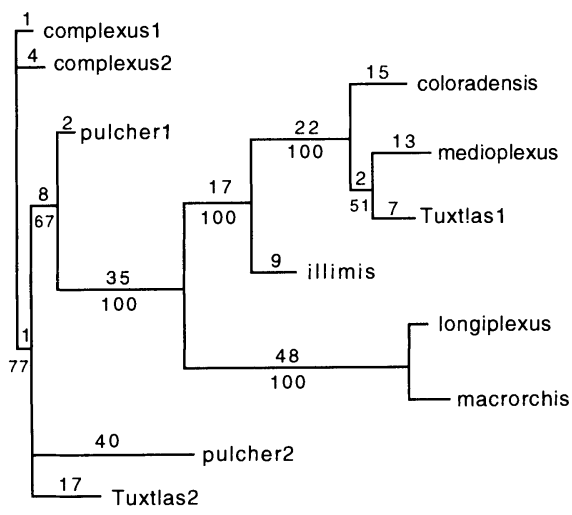

FIGURE 2. Most parsimonious unrooted trees (MPT) from the separate data sets. Values considering gaps as missing data in parentheses. (a) MPT from ITS1 sequences CI $=0.970(0.962)$; (b) majority rule consensus of 8 MPTs obtained from ITS2 sequences CI $=0.947$ (0.962); (c) majority rule consensus of 6 MPTs obtained from $28 \mathrm{~s} \mathrm{se}-$ quences $\mathrm{CI}=0.877$ (0.868). Bootstrap values $(1,000$ replicates) shown below the branches; branch length shown above.

atively large pharynx, and its host, salamanders of the genus Ambystoma Tschudi, 1832 (Bravo, 1943), the latter a circular criterion to use for distinguishing species (Brooks and McLennan, 1993). In the specimens we collected, the pharynx was not clearly larger than in the specimens of $H$. complexus from frogs from the same locality, and the pharyngeal glands, specially in unstained specimens, were no more distinct than those found in other digeneans. Separately sequenced specimens collected from salamanders represented 2 distinct genotypes: pulcher1 differs less (1.2\% variation) from $H$. complexus of $R$. montezumae in the same locality than did specimens of $H$. longiplexus from the U.S.A. and Mexico. Specimens of this genotype were likely an infection of $H$. complexus in Ambystoma

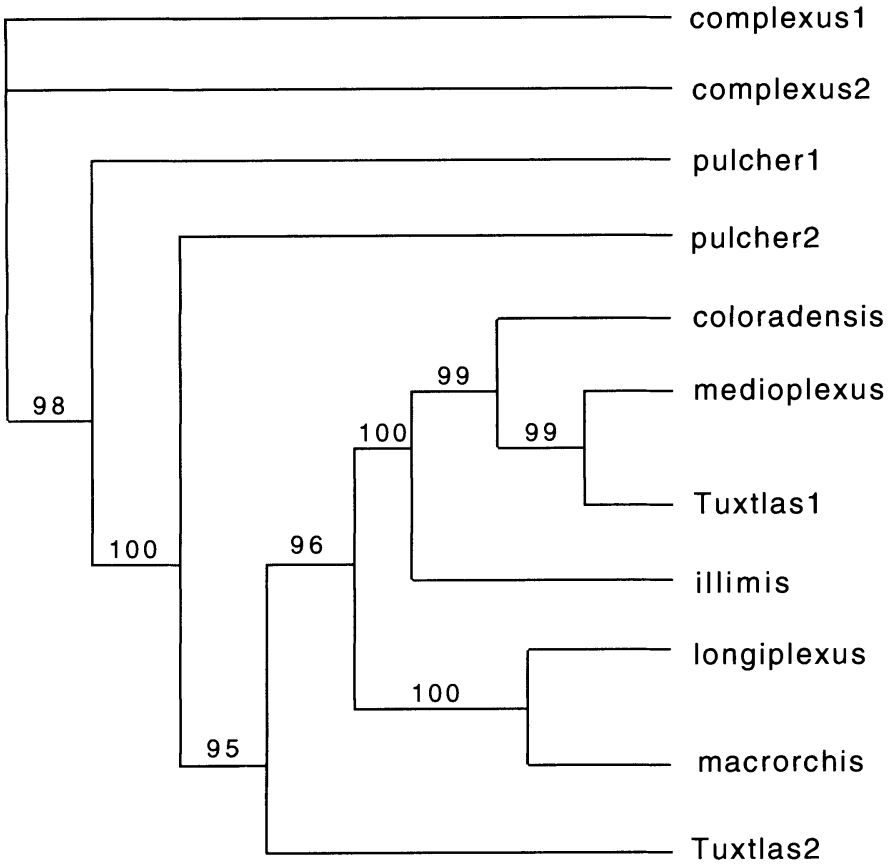

FIGURE 3. Most parsimonious unrooted tree obtained from the combined ITS1, ITS2, and 28s data sets; CI $=0.836$. Bootstrap values shown (1,000 replicates).

lermaensis Taylor, 1940. Pulcher2, by contrast, showed 5\% variation, with $H$. complexus, equivalent to that observed among many clearly differentiated morphospecies. We believe pulcher2, presumably the true $H$. pulcher, is a sibling species with $H$. complexus. If true, we believe that examination of additional material will allow us to discern morphological traits to differentiate them.

Finally, Tuxtlas 2 differs from $H$. complexus and $H$. pulcher in $5-8 \%$ of its sequence, indicating that it is also a distinct species in the complexus group. Further sampling in the area must be done to clarify the specific identity of this species.

Specimens designated as Tuxtlas 1 vary $2.0 \%$ from those of $H$. medioplexus in Nebraska. The low level of genetic variation indicates limited geographic differentiation, suggesting that the species should be continuously distributed at least from $\mathrm{Ne}-$ braska to Los Tuxtlas. Haematoloechus medioplexus has been collected in several different host species but most frequently in members of the $R$. pipiens, or leopard frog, clade in central and eastern U.S.A. and Canada. In accordance with the distribution ranges documented by Hillis et al. (1983) and Hillis (1988), published records listing $R$. pipiens as host may have been $R$. pipiens, Rana sphenocephala Cope, $1889, R$. blairi, or Rana berlandieri Baird, 1854. Since the recognition that the leopard frogs represent numerous different species, $H$. medioplexus has been reported in $R$. pipiens and $R$. blairi from Nebraska (Brooks, 1976). We have recently collected $H$. medioplexus in $R$. sphenocephala from Arkansas (D. Brooks, unpubl. obs.). Haematoloechus medioplexus has also been reported in Rana palustris, another member of the leopard frog clade in Massachusetts and Maine (Rankin, 1945; Bouchard, 1951).

Two members of the leopard frog clade occur in the Veracruz region, where los Tuxtlas is located. Rana berlandieri is distributed from Texas southward along the the east coast of Mex- 
ico to Veracruz; the northern part of its range overlaps with that of $R$. sphenocephala. Rana brownorum is distributed from Veracruz to Tabasco, Campeche, and Chiapas, Mexico (Frost, 1985).

In Mexico, $H$. medioplexus has been reported at low prevalence in $R$. vaillanti (this study) and Rana palmipes from Los Tuxtlas in Veracruz (Guillén-Hernández, 1992), and in $R$. montezumae from the Lerma wetlands and Lake Xochimilco (Caballero, 1941). Material from Lerma was not deposited in the CNHE and is not available for examination. We examined material from Xochimilco (CNHE 1191, 1770) and found that they do not belong to $H$. medioplexus. According to the arrangement of the uterine loops and the lack of acetabulum, they might belong to Haematoloechus iturbei Cordero and Vogelsang, 1939 or a closely related form. Further analysis of additional material will allow the identity of these specimens to be determined. Thus, it appears that in Mexico $H$. medioplexus occurs only along the eastern coast. We believe that $R$. vaillanti and $R$. palmipes Spix, 1824 from which $H$. medioplexus has been collected in Los Tuxtlas, are probably not the main hosts for this species of lung fluke in the region. First, both those frog species reach their northernmost extent in eastern Mexico, where $H$. medioplexus reaches it southernmost known distribution. Second, the typical Haematoloechus of $R$. palmipes is $H$. iturbei in South America, although this species was misidentified as $H$. medioplexus in Colombia (Uribe-Piedrahita, 1948). We have collected $H$. iturbei in $R$. palmipes from the Area de Conservación Guanacaste in northwestern Costa Rica, and it is clearly distinguishable from $H$. medioplexus by the lack of acetabulum and the unordered disposition of the uterus. We expect $R$. berlandieri to be the main host for $H$. medioplex$u s$ in los Tuxtlas as well as throughout its range in the eastern coast of Mexico.

Two species generally considered members of the $H$. complexus group, $H$. coloradensis and $H$. illimis, appear more closely related to $H$. medioplexus than to $H$. complexus in our analysis. They differ $2.7 \%$ and $4.2-5.1 \%$, respectively, from $H$. medioplexus, whereas they differ $10.3-10.6 \%$ and $10.3-10.5 \%$ from $H$. complexus. Haematoloechus coloradensis has generally been considered most similar to $H$. complexus (Kennedy [1981] suggested synonymizing them), from which it has been distinguished by having a relatively larger pharynx with respect to the oral sucker and a spinose tegument. We have already discussed the doubtful validity of the spined tegument as an informative character by itself. We found 2 morphological features, however, that are useful for distinguishing this species. The pharynx in all specimens is relatively larger and generally longer than the oral sucker than that of any members of the $H$. complexus group or of $H$. illimis or $H$. medioplexus. In addition, we discovered that the arrangement of the uterine loops is an informative character. Members of the $H$. complexus group and the $H$. medioplexus group lack longitudinal extracecal uterine loops, the plesiomorphic condition for plagiorchiform digeneans (Brooks et al., 1985). In members of the H. complexus group and in $H$. illimis, the postcecal uterine loops are not ordered into a well-differentiated ascending and a descending row, can overlap the ceca, and can occupy the total postcecal space. In $H$. coloradensis and $H$. medioplexus, however, the transverse uterine loops are ordered into a well-differentiated ascending and a descending row and occupy only intercecal space. These are the only 2 species of Haematoloechus presently known to exhibit this trait, which we conclude is a morphological synapomorphy linking them, thus corroborating the molecular data. Haematoloechus medioplexus further differs from $\boldsymbol{H}$. coloradensis by having an exremely small acetabulum, generally only $25 \%$ the width of the oral sucker, and by having extremely dense tegumental spination. These 2 species exhibit some degree of geographic differentiation. Haematoleochus medioplexus, as we have indicated, seems to be a species of the lowlands east of the Rocky Mountains down along to the eastern coastal area of Mexico, whereas $H$. coloradensis is a species of the western plateau in the U.S.A. (Colorado, Utah, Idaho, Nebraska) and central plateau in Mexico (Lake Pátzcuaro and Lerma wetlands), although both species occur in Nebraska (Brooks, 1976). Finally, both species inhabit primarily members of the leopard frog clade, with $H$. medioplexus known to occur in $R$. palustris, $R$. pipiens, $R$. blairi, and $R$. sphenocephala and presumed to occur in $R$. berlandieri, and $H$. coloradensis known to occur in $R$. pipiens, $R$. blairi, $R$. montezumae, and Rana dunni.

The sister species of $H$. medioplexus $+H$. coloradensis in this study is $H$. illimis, differing in $4.2-5.1 \%$ of its sequence. This species was described from $R$. montezumae in Lerma wetlands more than $50 \mathrm{yr}$ ago (Caballero, 1942b) and never collected again until now. Caballero (1942b) reported it from the lungs, but most of the specimens we collected were found in the eustachian tubes of the frogs, an unusual habitat for Haematoloechus. This species, generally considered in the complexus group, differs markedly from other members of the group, together with $H$. tumidus, by having lobed ovary and testes and several short extracecal uterine loops in the posterior half of the body. Caballero (1942b) also mentioned a large metraterm as a distinctive character for $H$. illimis. Molecular data support the exclusion of $\boldsymbol{H}$. illimis from the complexus group and its inclusion in the medioplexus group. Nevertheless, it differs from $H$. coloradensis and $H$. medioplexus in the arrangement of the uterus and in the shape of the ovary and testes. Morphological differences, together with the large amount of molecular variation with respect to $H$. coloradensis and $H$. medioplexus, suggest that it might be more closely related to other groups of species in the genus Haematoloechus. Further studies including other North American species might indicate its relation with other members of the genus.

This report has shown the merits and necessity of interpreting molecular data in the light of critical morphological evaluation to document the basic units of evolution and biodiversity: species. Finding substantial agreement between morphological and molecular data gives us hope that a robust phylogenetic hypothesis based on all available evidence (Kluge, 1989; Kluge and Wolf, 1993) can be produced for this fascinating group of digeneans. Given the geographic distribution and host range of Haematoloechus species, no doubt this group can become an important model system for historical ecological and parascript studies (Brooks and McLennan, 1991, 1993).

\section{ACKNOWLEDGMENTS}

This study was conducted at the Molecular Systematics Laboratory, Department of Zoology, University of Toronto and Royal Ontario Museum (ROM) and financed by Fundación 
UNAM, International Council for Canadian Studies Postdoctoral Fellowships and CONACYT proj. J27985N to V.L.-R., NSERC operating grant no. A7696 to D.R.B., and CONACYT proj.2676PN, PAPIIT proj. IN219198 to G.P.-P.L. Special thanks to Jinzhong Fu and Bob Murphy (ROM) and J. P. Laclette and M. García V. (Instituto de Investigaciones Biomédicas, UNAM) for their advice in DNA sequencing, to B. Moore, Skip Sterner, L. García Prieto, A. Jiménez R., B. Mendoza G., G. Pulido F., U. Razo M., C. Rosas V., and A. Sanchez A. for their help in field collections and 3 anonymous reviewers who contributed with valuable suggestions.

\section{LITERATURE CITED}

BARKER, S. C., AND D. BLAIR. 1996. Molecular phylogeny of Schistosoma species supports traditional groupings within the genus. Journal of Parasitology 82: 292-298.

, 一, A. R. GarretT, AND T. H. CRIBB. 1993. Utility of the D1 domain of nuclear 28s rRNA for phylogenetic inference in the Digenea. Systematic Parasitology 26: 181-188.

Blair, D., A. Campos, M. P. Cummings, and J. P. Laclette. 1996. Evolutionary biology of parasitic platyhelminths: The role of molecular phylogenetics. Parasitology Today 12: 66-71.

BOUCHARD, J. L. 1951. The platyhelminths parasitizing some northern Maine amphibia. Transactions of the American Microscopical Society 70: $245-250$.

Bowles, J., D. Blair, AND D. P. McManus. 1995. A molecular phylogeny of the genus Echinococcus. Parasitology 110: 317-328.

Bravo, H., M. 1943. Estudio sistemático de los tremátodos parásitos de los "ajolotes" de México I. Anales del Instituto de Biología Universidad Nacional Autónoma de México 14: 141-159.

BRooks, D. R. 1976. Parasites of amphibians of the Great Plains. II. Platyhelminths of amphibians in Nebraska. Bulletin of the University of Nebraska State Museum 10: 65-92.

- AND D. A. MCLENNAN. 1991. Phylogeny, ecology and behavior. A research program in comparative biology. University of Chicago Press, Chicago, Illinois, $434 \mathrm{p}$.

, AND - 1993. Parascript. Parasites and the language of evolution. Smithsonian Institution Press, Washington, D.C., 429 p. , R. T. O'GradY, AND D. R. GLEN. 1985. Phylogenetic analysis of the Digenea (Platyhelminthes: Cercomeria) with comments on their adaptive radiation. Canadian Journal of Zoology 63: 411-443.

Buckler, E. S. I., A. IpPolito, AND T. P. Holtsford. 1997. The evolution of ribosomal DNA: Divergent paralogues and phylogenetic implications. Genetics 145: 821-832.

Caballero, y C. E. 1941. Tremátodos de la Ciénaga de Lerma, Méx. Anales del Instituto de Biología Universidad Nacional Autónoma de México 12: 623-641.

- 1942a. Tremátodos de las ranas de la Ciénaga de Lerma, Estado de México. IV. Anales del Instituto de Biología Universidad Nacional Autónoma de México 13: 635-640.

-1942b. Tremátodos de las ranas de la Ciénaga de Lerma, Estado de México. II. Descripción de una nueva especie de Haematoloechus. Revista Brasileira de Biologia 2: 155-158.

- AND D. SOKOLOFF. 1934. Segunda contribución al conocimiento de la parasitología de Rana montezumae con un resumen, descripción de una nueva especie y clave del género Haematoloechus. Anales del Instituto de Biología Universidad Nacional Autónoma de México 5: 5-40.

Cabot, E. L., And A. T. Beckenbach. 1989. Simultaneous editing of multiple nucleic acid and protein sequences with ESEE. Comparative Applied Biology 5: 233-234.

CORT, W. W. 1915. North American frog lung flukes. Transactions of the American Microscopical Society 34: 203-240.

FLORES-VILleLA, O. 1993. Herpetofauna mexicana. Special Publication No. 17, Carnegie Museum of Natural History, Pittsburgh, Ohio, $73 \mathrm{p}$

Frost, D. R. 1985. Amphibian species of the world. A taxonomic and geographical reference. Allen Press, Lawrence, Kansas, 732 p.

GuILLÉN-HERNÁNDEZ, S. 1992. Comunidades de helmintos de algunos anuros de Los Tuxtlas, Veracruz. Tesis Maestría Facultad de Ciencias. UNAM, México City, México, 66 p.

HILlis, D. M. 1988. Systematics of the Rana pipiens complex: Puzzle and paradigm. Annual Review of Ecology and Systematics 19: 3963.

, AND T. M. DiXon. 1991. Ribosomal DNA: Molecular evolution and phylogenetic inference. Quarterly Review of Biology 66: 411453.

, J. S. Frost, AND D. A. Wright. 1983. Phylogeny and biogeography of the Rana pipiens complex: A biochemical evaluation. Systematic Zoology 32: 132-143.

- AND J. P. HUELSENBECK. 1992. Signal, noise and reliability in molecular phylogenetic analyses. Journal of Heredity 83: 189-195.

$\longrightarrow$, B. K. Mable, AND C. Moritz. 1996. Nucleic acids IV: Sequencing and cloning. In Molecular systematics, D. M. Hillis, C. Moritz, and B. K. Mable (eds.). Sinauer, Sunderland, Massachusetts. p. 321-383.

Kane, R. A., AND D. Rollinson. 1994. Repetitive sequences in the ribosomal DNA internal transcribed spacer of Schistosoma haematobium, Schistosoma intercalatum and Schistosoma mattheei. Molecular and Biochemical Parasitology 63: 153-156.

KENNEDY, M. J. 1980a. Host-induced variations in Haematoloechus buttensis (Trematoda: Haematoloechidae). Canadian Journal of Zoology 58: 427-442.

- 1980b. Geographical variation in some representatives of Haematoloechus, Looss, 1899 (Trematoda: Haematoloechidae) from Canada and the United States. Canadian Journal of Zoology 58: $1151-1167$.

- 1981. A revision of species of the genus Haematoloechus Looss, 1899 (Trematoda: Haematoloechidae) from Canada and the United States. Canadian Journal of Zoology 59: 1836-1846.

KLUGE, A. G. 1989. A concern for evidence and phylogenetic hypothesis of relationships among Epicrates (Boidae, Serpentes). Systematic Zoology 38: 7-25.

, AND A. J. WOLF. 1993. Cladistics: What's in a word? Cladistics 9: $183-199$.

Krull, W. H. 1932. Studies on the life history of Pneumobites longiplexus (Stafford). Zoologischer Anzeiger 99: 231-239.

- 1933. Studies on the life history of a frog lung fluke, Haematoloechus complexus (Seely, 1906) Krull, n. comb. Parasitenkunde 6: 192-206.

LitTLEwood, D. T., AND D. A. Johnston. 1995. Molecular phylogenetics of the four Schistosoma species groups determined with partial $28 \mathrm{~S}$ ribosomal RNA gene sequences. Parasitology 111: 167-175.

LEÓN-RĖGAGNON, V. 1992. Fauna helmintológica de algunos vertebrados acuáticos de la Ciénaga de Lerma, Estado de México. Anales del Instituto de Biología Universidad Nacional Autónoma de México 63: $151-153$.

Luton, K., D. WALKeR, AND D. Blair. 1992. Comparisons of ribosomal internal transcribed spacers from two congeneric species of flukes (Platyhelminthes: Trematoda: Digenea). Molecular and Biochemical Parasitology 56: 323-328.

MadDISON W. P., AND D. R. MAdDISON. 1992. MacClade, Version 3.0. Sinauer Sunderland, Massachusetts.

MANTER, H. W. 1938. A collection of trematodes from Florida amphibia. Transactions of the American Microscopical Society 57: 26-38.

MartíneZ V., J. M. 1969. Parásitos de algunos anfibios colectados en diferentes áreas de los municipios de Escobedo, Pesquería, Santiago, Nuevo León, México. Tesis Facultad de Ciencias Biológicas. Universidad Autónoma de Nuevo León, Monterrey, México, 51 p.

MCMANus, D. P., AND J. Bowles. 1996. Molecular genetic approaches to parasite identification: Their value in diagnostic parasitology and systematics. International Journal for Parasitology 26: 687-704.

Morgan, J. A. T., AND D. BLAIR. 1995. Nuclear rDNA ITS sequence variation in the trematode genus Echinosotoma: An aid to establishing relationships within the 37-spine group. Parasitology 111: 609-615.

PAlumBI, S. R. 1996. Nucleic acids II: The polymerase chain reaction. In Molecular systematics, D. M. Hillis, C. Moritz, and B. K. Mable (eds.). Sinauer, Sunderland, Massachusetts, p. 205-247

Prokopic, J., AND K. Krivanec. 1974. Trematodes of the genus Hae- 
matoloechus Looss, and their variability. Helminthologia 15: 779 802.

Pulido F., G. 1994. Helmintos de Rana dunni especie endémica del lago de Pátzcuaro, Michoacán, México. Anales del Instituto de Biología Universidad Nacional Autónoma de México 65: 205-207.

RANKIN, J. S. 1945. An ecological study of the helminth parasites of amphibians and reptiles of western Massachusetts and vicinity. Journal of Parasitology 31: 142-150.

Rollinson, D., T. K. Walker, R. J. Knowles, and A. J. G. Simpson. 1990. Identification of schistosome hybrids and larval parasites using rRNA probes. Systematic Parasitology 15: 65-73.

STAFFORD, J. 1902. On the american representatives of Distomum var- iegatum. Zoologische Jahrbuecher Abteilung fuer Systematik Oekologie und Geographie der Tiere 16: 895-912.

SwOFFORD, D. L. 1993. PAUP: Phylogenetic analysis using parsimony, Version 3.1.1. Illinois Natural History Survey, Champaign, Illinois Thompson, J. D., D. G. Higgins, and T. J. Gibson. 1994. Clustal W: Improving the sensitivity of progressive multiple sequence alignment through sequence weighting, position-specific gap penalties and weight matrix choice. Nucleic Acids Research 22: 4673-4680.

URIBE-Piedrahita, C. 1948. Contribuciones al estudio de la parasitología en Colombia II. Caldasia, Bogotá 5: 211-219.

WeINS, J. J. 1998. Combining data sets with different phylogenetic histories. Systematic Biology 47: 568-581. 